\title{
Dykstra's Algorithm for the Optimal Approximate Symmetric Positive Semidefinite Solution of a Class of Matrix Equations*
}

\author{
Chunmei Li", Xuefeng Duan, Zhuling Jiang \\ College of Mathematics and Computational Science, Guilin University of Electronic Technology, Guilin, China \\ Email: "lengyue123@@126.com
}

Received 4 December 2015; accepted 4 March 2016; published 7 March 2016

Copyright (C) 2016 by authors and Scientific Research Publishing Inc.

This work is licensed under the Creative Commons Attribution International License (CC BY). http://creativecommons.org/licenses/by/4.0/

(c) (i) Open Access

\begin{abstract}
Dykstra's alternating projection algorithm was proposed to treat the problem of finding the projection of a given point onto the intersection of some closed convex sets. In this paper, we first apply Dykstra's alternating projection algorithm to compute the optimal approximate symmetric positive semidefinite solution of the matrix equations $A X B=E, C X D=F$. If we choose the initial iterative matrix $X_{0}=0$, the least Frobenius norm symmetric positive semidefinite solution of these matrix equations is obtained. A numerical example shows that the new algorithm is feasible and effective.
\end{abstract}

\section{Keywords}

Matrix Equation, Dykstra's Alternating Projection Algorithm, Optimal Approximate Solution, Least Norm Solution

\section{Introduction}

Throughout this paper, we use $R^{m \times n}$ and $S P^{n \times n}$ to stand for the set of $m \times n$ real matrices and $n \times n$ symmetric positive semidefinite matrices, respectively. We denote the transpose and Moore-Penrose generalized inverse of the matrix $\boldsymbol{A}$ by $\boldsymbol{A}^{\mathrm{T}}$ and $\boldsymbol{A}^{+}$, respectively. The symbol $I_{n}$ stands for $n \times n$ identity matrix. For

\footnotetext{
${ }^{*}$ The work was supported by National Natural Science Foundation of China (No.11561015; 11261014; 11301107), Natural Science Foundation of Guangxi Province (No.2012GXNSFBA053006; 2013GXNSFBA019009).

"Corresponding author.

How to cite this paper: Li, C.M., Duan, X.F. and Jiang, Z.L. (2016) Dykstra's Algorithm for the Optimal Approximate Symmetric Positive Semidefinite Solution of a Class of Matrix Equations. Advances in Linear Algebra \& Matrix Theory, 6, 1-10. http://dx.doi.org/10.4236/alamt.2016.61001
} 
$\boldsymbol{A}, \boldsymbol{B} \in R^{m \times n}, \quad\langle\boldsymbol{A}, \boldsymbol{B}\rangle=\operatorname{trace}\left(\boldsymbol{B}^{\mathrm{T}} \boldsymbol{A}\right)$ denotes the inner product of the matrix $\boldsymbol{A}$ and $\boldsymbol{B}$. The induced norm is the so-called Frobenius norm, that is, $\|\boldsymbol{A}\|=\langle\boldsymbol{A}, \boldsymbol{A}\rangle^{1 / 2}$, then $R^{m \times n}$ is a real Hilbert space. In order to develop this paper, we need to give the following definition.

Definition 1.1. [1] Let $M$ be a closed convex subset in a real Hilbert space $H$ and $u$ be a point in $H$, then the point in $M$ nearest to $u$ is called the projection of $u$ onto $M$ and denoted by $P_{M}(u)$, that is to say, $P_{M}(u)$ is the solution of the following minimization problem

$$
\min _{x \in M}\|x-u\|
$$

i.e.

$$
\left\|P_{M}(u)-u\right\|=\min _{x \in M}\|x-u\| .
$$

In this paper, we consider the matrix equations

$$
A X B=E, \quad C X D=F,
$$

and their matrix nearness problem.

Problem I. Given matrices $\boldsymbol{A} \in R^{p \times n}, \quad \boldsymbol{B} \in R^{n \times q}, \quad \boldsymbol{C} \in R^{s \times n}, \quad \boldsymbol{D} \in R^{n \times t}, \quad \boldsymbol{E} \in R^{p \times q}, \quad \boldsymbol{F} \in R^{s \times t}$ and $\overline{\boldsymbol{X}} \in R^{n \times n}$, find $\hat{\boldsymbol{X}} \in \Omega$ such that

$$
\|\hat{\boldsymbol{X}}-\overline{\boldsymbol{X}}\|=\min _{X \in \Omega}\|\boldsymbol{X}-\overline{\boldsymbol{X}}\|,
$$

where

$$
\Omega=\left\{\boldsymbol{X} \in S P^{n \times n} \mid \boldsymbol{A X} \boldsymbol{B}=\boldsymbol{E}, \boldsymbol{C X} \boldsymbol{D}=\boldsymbol{F}\right\} .
$$

Obviously, $\Omega$ is the symmetric positive semidefinite solution set of the matrix equations (1.3). It is easy to verify that $\Omega$ is a closed convex set, then the solution $\hat{\boldsymbol{X}}$ of Problem I is unique. In this paper, the unique solution $\hat{\boldsymbol{X}}$ is called the optimal approximate symmetric positive semidefinite solution of Equation (1.3). In particular, if $\overline{\boldsymbol{X}}=0$, then the solution $\hat{\boldsymbol{X}}$ of Problem I is just the least Frobenius norm symmetric positive semidefinite solution of the matrix equations (1.3).

This kind of matrix nearness problem occurs frequently in experimental design, see for instance [2] [3]. Here $\overline{\boldsymbol{X}}$ may be obtained from experiments, but not satisfy Equation (1.3). The nearest matrix $\hat{\boldsymbol{X}}$ satisfies Equation (1.3) and is nearest to the given matrix $\overline{\boldsymbol{X}}$. Up to now, Equation (1.3) and their matrix nearness problem I have been extensively studied for the past 40 or more years. Navarra-Odell-Young [4] and Wang [5] gave necessary and sufficient conditions for Equation (1.3) having a solution and presented the expression for a general solution. By the projection theorem and matrix decompositions, Liao-Lei-Yuan [6] [7] gave some analytical expressions of the optimal approximate least square symmetric solution of Equation (1.3). ShengChen [8] presented an efficient iterative method to compute the optimal approximate solution for the matrix equations (1.3). Ding-Liu-Ding [9] considered the unique solution of Equation (1.3) and used gradient based iterative algorithm to compute the unique solution. Peng-Hu-Zhang [10] and Chen-Peng-Zhou [11] proposed some iterative methods to compute the symmetric solutions and optimal approximate symmetric solution of Equation (1.3). The (least square) solution and the optimal approximate (least square) solution of Equation (1.3), which is constrained as bisymmetric, reflexive, generalized reflexive, generalized centro-symmetric, were studied in [11]-[17]. Nevertheless, to the best of our knowledge, the optimal approximate solution of Equation (1.3), which is constrained as symmetric positive semidefinite, (i.e. Problem I) has not been solved. The difficulty of Problem I lies in how to characterize the convex set $\Omega$. In this paper, we first divided the set $\Omega$ into three sets $\Omega_{1}, \Omega_{2}, \Omega_{3}$, and then adopt alternating projections to overcome the difficulty.

Dykstra's alternating projection algorithm was proposed by Dykstra [18] to treat the problem of finding the projection of a given point onto the intersection of some closed convex sets. It is based on a clear modification of the classical alternating projection algorithm first proposed by Von Neumann [19], and studied later by Cheney and Goldstein [20]. For an application of Dykstra's alternating projection algorithm to compute the nearest diagonally dominant matrix see [21]. For a complete survey on Dykstra's alternation projection algorithm and applications see Deutsch [22]. 
In this paper, we propose a new algorithm to compute the optimal approximate symmetric positive semidefinite solution of Equation (1.3). We state Problem I as the minimization of a convex quadratic function over the intersection of three closed convex sets in the vector space $R^{n \times n}$. From this point of view, Problem I can be solved by the Dykstra's alternating projection algorithm. If we choose the initial iterative matrix $\boldsymbol{X}_{0}=0$, the least Frobenius norm symmetric positive semidefinite solution of the matrix equations $\boldsymbol{A X \boldsymbol { B }}=\boldsymbol{E}, \boldsymbol{C X \boldsymbol { D }}=\boldsymbol{F}$ is obtained. In the end, we use a numerical example to show that the new algorithm is feasible and effective.

\section{Dykstra's Algorithm for Solving Problem I}

In this section, we apply Dykstra's alternating projection algorithm to compute the optimal approximate symmetric positive semidefinite solution of Equation (1.3). We first introduce Dykstra's alternating projection algorithm and its convergence theorem.

In order to find the projection of a given point onto the intersection of a finite number of closed convex sets $C_{1}, C_{2}, \cdots, C_{n}$, Dykstra [18] proposed Dykstra's alternating projection algorithm which can be stated as follows. This algorithm can be also seen in [1] [23]-[25].

\section{Dykstra's Algorithm 2.1}

1) Given the initial value $x_{0}$;

2) Set $x_{n}^{(0)}=x_{0}, I_{i}^{(0)}=0, i=1,2, \cdots, n$

3) For $k=1,2,3, \cdots$

$$
x_{0}^{(k)}=x_{n}^{(k-1)}
$$

For $i=1,2, \cdots, n$

$$
\begin{aligned}
& x_{i}^{(k)}=P_{C_{i}}\left(x_{i-1}^{(k)}-I_{i}^{(k-1)}\right), \\
& I_{i}^{(k)}=x_{i}^{(k)}-\left(x_{i-1}^{(k)}-I_{i}^{(k-1)}\right)
\end{aligned}
$$

End

End

The utility of Dykstra's algorithm 2.1 is based on the following theorem (see [23]-[25] and the references therein).

Lemma 2.1. ([23], Theorem 2) Let $C_{1}, C_{2}, \cdots, C_{n}$ be closed convex subsets of a real Hilbert space $H$ such that $C_{1} \cap C_{2} \cap \cdots \cap C_{n} \neq \varnothing$. For any $i=1,2, \cdots, n$ and any $x_{0} \in H$, the sequences $\left\{x_{i}^{(k)}\right\}$ generated by Dykstra's algorithm 2.1 converge to $P_{C_{1} \cap C_{1} \cap \cdots \cap C_{n}}\left(x_{0}\right)$, that is,

$$
x_{i}^{(k)} \rightarrow P_{C_{1} \cap C_{1} \cap \cdots \cap C_{n}}\left(x_{0}\right), i=1,2, \cdots, n, k \rightarrow+\infty .
$$

Now we begin to use Dykstra's algorithm 2.1 to solve Problem I. Firstly, we define three sets

$$
\Omega_{1}=\left\{\boldsymbol{X} \in R^{n \times n} \mid \boldsymbol{A X} \boldsymbol{B}=\boldsymbol{E}\right\}, \Omega_{2}=\left\{\boldsymbol{X} \in R^{n \times n} \mid \boldsymbol{C X} \boldsymbol{D}=\boldsymbol{F}\right\} \text { and } \Omega_{3}=\boldsymbol{S} P^{n \times n} .
$$

It is easy to know that $\Omega=\Omega_{1} \cap \Omega_{2} \cap \Omega_{3}$, and if the set $\Omega$ is nonempty, then

$$
\Omega=\Omega_{1} \cap \Omega_{2} \cap \Omega_{3} \neq \varnothing .
$$

On the other hand, it is easy to verify that $\Omega_{1}, \Omega_{2}$ and $\Omega_{3}$ are closed convex subsets of the real Hilbert space $R^{n \times n}$.

After defining the sets $\Omega_{1}, \Omega_{2}$ and $\Omega_{3}$, Problem I can be rewritten as finding $\hat{\boldsymbol{X}} \in \Omega=\Omega_{1} \cap \Omega_{2} \cap \Omega_{3}$, such that

$$
\|\hat{\boldsymbol{X}}-\overline{\boldsymbol{X}}\|=\min _{X \in \Omega_{1} \cap \Omega_{2} \cap \Omega_{3}}\|\boldsymbol{X}-\overline{\boldsymbol{X}}\| .
$$

By Definition 1.1 and noting that the equalities (2.2) and (1.2), it is easy to find that

$$
\hat{\boldsymbol{X}}=P_{\Omega_{1} \cap \Omega_{2} \cap \Omega_{3}}(\overline{\boldsymbol{X}}) .
$$


Therefore, Problem I can be converted equivalently into finding the projection $P_{\Omega_{1} \cap \Omega_{2} \cap \Omega_{3}}(\overline{\boldsymbol{X}})$. Now we will use Dykstra's algorithm 2.1 to compute the projection $P_{\Omega_{1} \cap \Omega_{2} \cap \Omega_{3}}(\overline{\boldsymbol{X}})$. By (2.3), we can get the optimal approximate symmetric positive semidefinite solution $\hat{\boldsymbol{X}}$ of the matrix equations (1.3).

We can see that the key problems to realize Dykstra's algorithm 2.1 are how to compute the projections $P_{\Omega_{1}}(Z), P_{\Omega_{2}}(Z)$ and $P_{\Omega_{3}}(Z)$ of a matrix $Z$ onto $\Omega_{1}, \Omega_{2}$ and $\Omega_{3}$, respectively. Such problems are perfectly solvable in the following theorems.

Theorem 2.1. Suppose that the set $\Omega_{1}$ is nonempty. For a given $n \times n$ matrix $\mathbf{Z}$, we have

$$
P_{\Omega_{1}}(Z)=Z+A^{+}(E-A Z B) B^{+} .
$$

Proof. By Definition 1.1, we know that the projection $P_{\Omega_{1}}(Z)$ is the solution of the following minimization problem

$$
\min _{X \in \Omega_{1}}\|\boldsymbol{X}-\boldsymbol{Z}\|
$$

Now we begin to solve the minimization problem (2.4). We first characterize the solution set $\Omega_{1}$, and then find $\hat{\boldsymbol{X}}$ such that (2.4) holds. Noting that the set $\Omega_{1}$ is a closed convex set, then the minimization problem (2.4) has a unique solution. Hence $\hat{\boldsymbol{X}}=P_{\Omega_{1}}(Z)$. The singular value decomposition of the matrices $\boldsymbol{A}$ and $\boldsymbol{B}$ are given by

$$
\boldsymbol{A}=\boldsymbol{U}\left(\begin{array}{ll}
\Gamma & 0 \\
0 & 0
\end{array}\right) \boldsymbol{V}^{\mathrm{T}}, \boldsymbol{B}=\tilde{\boldsymbol{U}}\left(\begin{array}{cc}
W & 0 \\
0 & 0
\end{array}\right) \tilde{\boldsymbol{V}}^{\mathrm{T}},
$$

where $\boldsymbol{U}=\left(\boldsymbol{U}_{1}, \boldsymbol{U}_{2}\right) \in R^{p \times p}, \quad \boldsymbol{V}=\left(\boldsymbol{V}_{1}, \boldsymbol{V}_{2}\right) \in \boldsymbol{R}^{n \times n}$ are orthogonal matrices, $\boldsymbol{U}_{1} \in \boldsymbol{R}^{p \times r}, \quad \boldsymbol{V}_{1} \in \boldsymbol{R}^{n \times r}$, $r=\operatorname{rank}(\boldsymbol{A}), \tilde{\Gamma}=\operatorname{diag}\left(t_{1}, t_{2}, \cdots, t_{r}\right)$ and $\tilde{\boldsymbol{U}}=\left(\tilde{\boldsymbol{U}}_{1}, \tilde{\boldsymbol{U}}_{2}\right) \in R^{n \times n}, \quad \tilde{\boldsymbol{V}}=\left(\tilde{\boldsymbol{V}}_{1}, \tilde{\boldsymbol{V}}_{2}\right) \in \boldsymbol{R}^{q \times q}$ are orthogonal matrices, $\tilde{U}_{1} \in R^{n \times s}, \quad \tilde{V}_{1} \in R^{q \times s}, \quad s=\operatorname{rank}(\boldsymbol{B}), \quad \boldsymbol{W}=\operatorname{diag}\left(w_{1}, w_{2}, \cdots, w_{s}\right)$. According to the definition of the Moore-Penrose generalized inverse of a matrix, we have

$$
\boldsymbol{A}^{+}=\boldsymbol{V}\left(\begin{array}{cc}
\Gamma^{-1} & 0 \\
0 & 0
\end{array}\right) \boldsymbol{U}^{\mathrm{T}}, \boldsymbol{B}^{+}=\tilde{\boldsymbol{V}}\left(\begin{array}{cc}
\boldsymbol{W}^{-1} & 0 \\
0 & 0
\end{array}\right) \tilde{\boldsymbol{U}}^{\mathrm{T}},
$$

and

$$
\boldsymbol{A}^{+} \boldsymbol{A}=\boldsymbol{V}_{1} \boldsymbol{V}_{1}^{\mathrm{T}}, \boldsymbol{B} \boldsymbol{B}^{+}=\tilde{\boldsymbol{U}}_{1} \tilde{\boldsymbol{U}}_{1}^{\mathrm{T}}, \boldsymbol{V}_{1} \boldsymbol{V}_{1}^{\mathrm{T}}+\boldsymbol{V}_{2} \boldsymbol{V}_{2}^{\mathrm{T}}=\boldsymbol{I}, \tilde{\boldsymbol{U}}_{1} \tilde{\boldsymbol{U}}_{1}^{\mathrm{T}}+\tilde{\boldsymbol{U}}_{2} \tilde{\boldsymbol{U}}_{2}^{\mathrm{T}}=\boldsymbol{I} .
$$

Substituting (2.5) into the matrix equation $\boldsymbol{A X B}=\boldsymbol{E}$, we obtain

$$
\boldsymbol{U}\left(\begin{array}{ll}
\boldsymbol{\Gamma} & 0 \\
0 & 0
\end{array}\right) \boldsymbol{V}^{\mathrm{T}} \boldsymbol{X} \tilde{\boldsymbol{U}}\left(\begin{array}{cc}
\boldsymbol{W} & 0 \\
0 & 0
\end{array}\right) \tilde{\boldsymbol{V}}^{\mathrm{T}}=\boldsymbol{E}
$$

which implies

$$
\left(\begin{array}{ll}
\Gamma & 0 \\
0 & 0
\end{array}\right) \boldsymbol{V}^{\mathrm{T}} \boldsymbol{X} \tilde{\boldsymbol{U}}\left(\begin{array}{cc}
\boldsymbol{W} & 0 \\
0 & 0
\end{array}\right)=\boldsymbol{U}^{T} \boldsymbol{E} \tilde{\boldsymbol{V}}
$$

Let

$$
\boldsymbol{V}^{\mathrm{T}} \boldsymbol{X} \tilde{\boldsymbol{U}}=\left(\begin{array}{ll}
\boldsymbol{X}_{11} & \boldsymbol{X}_{12} \\
\boldsymbol{X}_{21} & \boldsymbol{X}_{22}
\end{array}\right), \boldsymbol{U}^{\mathrm{T}} \boldsymbol{E} \tilde{\boldsymbol{V}}=\left(\begin{array}{c}
\boldsymbol{U}_{1}^{\mathrm{T}} \\
\boldsymbol{U}_{2}^{\mathrm{T}}
\end{array}\right) \boldsymbol{E}\left(\begin{array}{ll}
\tilde{\boldsymbol{V}}_{1} & \tilde{\boldsymbol{V}}_{2}
\end{array}\right)=\left(\begin{array}{cc}
\boldsymbol{U}_{1}^{\mathrm{T}} \boldsymbol{E} \tilde{\boldsymbol{V}}_{1} & \boldsymbol{U}_{1}^{\mathrm{T}} \boldsymbol{E} \tilde{\boldsymbol{V}}_{2} \\
\boldsymbol{U}_{2}^{\mathrm{T}} \boldsymbol{E} \tilde{\boldsymbol{V}}_{1} & \boldsymbol{U}_{2}^{\mathrm{T}} \boldsymbol{E} \tilde{\boldsymbol{V}}_{2}
\end{array}\right) .
$$

Then the matrix equation $\boldsymbol{A X} \boldsymbol{B}=\boldsymbol{E}$ can be equivalently written as

$$
\left(\begin{array}{ll}
\boldsymbol{\Gamma} & 0 \\
0 & 0
\end{array}\right)\left(\begin{array}{ll}
\boldsymbol{X}_{11} & \boldsymbol{X}_{12} \\
\boldsymbol{X}_{21} & \boldsymbol{X}_{22}
\end{array}\right)\left(\begin{array}{cc}
\boldsymbol{W} & 0 \\
0 & 0
\end{array}\right)=\left(\begin{array}{lll}
\boldsymbol{U}_{1}^{\mathrm{T}} \boldsymbol{E} \tilde{\boldsymbol{V}}_{1} & \boldsymbol{U}_{1}^{\mathrm{T}} \boldsymbol{E} \tilde{\boldsymbol{V}}_{2} \\
\boldsymbol{U}_{2}^{\mathrm{T}} \boldsymbol{E} \tilde{\boldsymbol{V}}_{1} & \boldsymbol{U}_{2}^{\mathrm{T}} \boldsymbol{E} \tilde{\boldsymbol{V}}_{2}
\end{array}\right),
$$

which implies that 


$$
\begin{gathered}
\boldsymbol{\Gamma} \boldsymbol{X}_{11} \boldsymbol{W}=\boldsymbol{U}_{1}^{\mathrm{T}} \boldsymbol{E} \tilde{\boldsymbol{V}}_{1}, \\
0=\boldsymbol{U}_{1}^{\mathrm{T}} \boldsymbol{E} \tilde{\boldsymbol{V}}_{2}, \\
0=\boldsymbol{U}_{2}^{\mathrm{T}} \boldsymbol{E} \tilde{\boldsymbol{V}}_{1}, \\
0=\boldsymbol{U}_{2}^{\mathrm{T}} \boldsymbol{E} \tilde{\boldsymbol{V}}_{2} .
\end{gathered}
$$

By (2.8) we have

$$
\boldsymbol{X}_{11}=\Gamma^{-1} \boldsymbol{U}_{1}^{\mathrm{T}} \boldsymbol{E} \tilde{\boldsymbol{V}}_{1} \boldsymbol{W}^{-1}
$$

Noting that the set $\Omega_{1}$ is nonempty, by (2.5) it is easy to verify that (2.9), (2.10) and (2.11) are identical equations. Hence the general solutions of the matrix equation $\boldsymbol{A X B}=\boldsymbol{E}$ can be expressed as

$$
\boldsymbol{X}=\boldsymbol{V}\left(\begin{array}{ll}
\boldsymbol{X}_{11} & \boldsymbol{X}_{12} \\
\boldsymbol{X}_{21} & \boldsymbol{X}_{22}
\end{array}\right) \tilde{\boldsymbol{U}}^{\mathrm{T}}=\boldsymbol{V}\left(\begin{array}{cc}
\boldsymbol{\Gamma}^{-1} \boldsymbol{U}_{1}^{\mathrm{T}} \boldsymbol{E} \tilde{\boldsymbol{V}}_{1} \boldsymbol{W}^{-1} & \boldsymbol{X}_{12} \\
\boldsymbol{X}_{21} & \boldsymbol{X}_{22}
\end{array}\right) \tilde{\boldsymbol{U}}^{T},
$$

where $\boldsymbol{X}_{12} \in R^{r \times(n-s)}, \boldsymbol{X}_{21} \in R^{(n-r) \times s}, \quad \boldsymbol{X}_{22} \in R^{(n-r) \times(n-s)}$ are arbitrary, which implies that the entries of the set $\Omega_{1}$ can be stated as (2.12).

Consequently,

$$
\begin{aligned}
\|\boldsymbol{X}-\boldsymbol{Z}\| & =\left\|\boldsymbol{V}\left(\begin{array}{cc}
\boldsymbol{\Gamma}^{-1} \boldsymbol{U}_{1}^{\mathrm{T}} \boldsymbol{E} \tilde{\boldsymbol{V}}_{1} \boldsymbol{W}^{-1} & \boldsymbol{X}_{12} \\
\boldsymbol{X}_{21} & \boldsymbol{X}_{22}
\end{array}\right) \tilde{\boldsymbol{U}}^{\mathrm{T}}-\boldsymbol{Z}\right\| \\
& =\left\|V\left(\left[\begin{array}{cc}
\Gamma^{-1} \boldsymbol{U}_{1}^{\mathrm{T}} \boldsymbol{E} \tilde{\boldsymbol{V}}_{1} \boldsymbol{W}^{-1} & \boldsymbol{X}_{12} \\
\boldsymbol{X}_{21} & \boldsymbol{X}_{22}
\end{array}\right)-\boldsymbol{V}^{\mathrm{T}} \boldsymbol{Z} \tilde{\boldsymbol{U}}\right] \tilde{\boldsymbol{U}}^{\mathrm{T}}\right\| \\
& =\left\|\left(\begin{array}{cc}
\boldsymbol{\Gamma}^{-1} \boldsymbol{U}_{1}^{\mathrm{T}} \boldsymbol{E} \tilde{\boldsymbol{V}}_{1} \boldsymbol{W}^{-1} & \boldsymbol{X}_{12} \\
\boldsymbol{X}_{21} & \boldsymbol{X}_{22}
\end{array}\right)-\left(\begin{array}{cc}
\boldsymbol{V}_{1}^{\mathrm{T}} \boldsymbol{Z} \tilde{\boldsymbol{U}}_{1} & \boldsymbol{V}_{1}^{\mathrm{T}} \boldsymbol{Z} \tilde{\boldsymbol{U}}_{2} \\
\boldsymbol{V}_{2}^{\mathrm{T}} \boldsymbol{Z} \tilde{\boldsymbol{U}}_{1} & \boldsymbol{V}_{2}^{\mathrm{T}} \boldsymbol{Z} \tilde{\boldsymbol{U}}_{2}
\end{array}\right)\right\| \\
& =\left\|\left(\begin{array}{cc}
\Gamma^{-1} \boldsymbol{U}_{1}^{\mathrm{T}} \boldsymbol{E} \tilde{\boldsymbol{V}}_{1} \boldsymbol{W}^{-1}-\boldsymbol{V}_{1}^{\mathrm{T}} \boldsymbol{Z} \tilde{\boldsymbol{U}}_{1} & \boldsymbol{X}_{12}-\boldsymbol{V}_{1}^{\mathrm{T}} \boldsymbol{Z} \tilde{\boldsymbol{U}}_{2} \\
\boldsymbol{X}_{21}-\boldsymbol{V}_{2}^{\mathrm{T}} \boldsymbol{Z} \tilde{\boldsymbol{U}}_{1} & \boldsymbol{X}_{22}-\boldsymbol{V}_{2}^{\mathrm{T}} \boldsymbol{Z} \tilde{\boldsymbol{U}}_{2}
\end{array}\right)\right\| .
\end{aligned}
$$

By (2.13) we know that $\|\boldsymbol{X}-\boldsymbol{Z}\|=\min _{X \in \Omega_{1}}$ if and only if

$$
\boldsymbol{X}_{12}{ }^{X \in \Omega_{1}}=\boldsymbol{V}_{1}^{\mathrm{T}} \boldsymbol{Z} \tilde{\boldsymbol{U}}_{2}, \boldsymbol{X}_{21}=\boldsymbol{V}_{2}^{\mathrm{T}} \boldsymbol{Z} \tilde{\boldsymbol{U}}_{1}, \boldsymbol{X}_{22}=\boldsymbol{V}_{2}^{\mathrm{T}} \boldsymbol{Z} \tilde{\boldsymbol{U}}_{2} .
$$

Therefore, the solution of the minimization problem (2.4) is

$$
\hat{\boldsymbol{X}}=P_{\Omega_{1}}(\boldsymbol{Z})=\boldsymbol{V}\left(\begin{array}{cc}
\boldsymbol{\Gamma}^{-1} \boldsymbol{U}_{1}^{\mathrm{T}} \boldsymbol{E} \tilde{\boldsymbol{V}}_{1} \boldsymbol{W}^{-1} & \boldsymbol{V}_{1}^{\mathrm{T}} \boldsymbol{Z} \tilde{\boldsymbol{U}}_{2} \\
\boldsymbol{V}_{2}^{\mathrm{T}} \boldsymbol{Z} \tilde{\boldsymbol{U}}_{1} & \boldsymbol{V}_{2}^{\mathrm{T}} \boldsymbol{Z} \tilde{\boldsymbol{U}}_{2}
\end{array}\right) \tilde{\boldsymbol{U}}^{\mathrm{T}} .
$$

Combining (2.14) and (2.5)-(2.7), we have

$$
\begin{aligned}
P_{\Omega_{1}}(\boldsymbol{Z}) & =\boldsymbol{V}\left(\begin{array}{cc}
\boldsymbol{\Gamma}^{-1} \boldsymbol{U}_{1}^{\mathrm{T}} \boldsymbol{E} \tilde{\boldsymbol{V}}_{1} \boldsymbol{W}^{-1} & \boldsymbol{V}_{1}^{\mathrm{T}} \boldsymbol{Z} \tilde{\boldsymbol{U}}_{2} \\
\boldsymbol{V}_{2}^{\mathrm{T}} \boldsymbol{Z} \tilde{\boldsymbol{U}}_{1} & \boldsymbol{V}_{2}^{\mathrm{T}} \boldsymbol{Z} \tilde{\boldsymbol{U}}_{2}
\end{array}\right) \tilde{\boldsymbol{U}}^{\mathrm{T}} \\
& =\left(\begin{array}{ll}
\boldsymbol{V}_{1} & \boldsymbol{V}_{2}
\end{array}\right)\left(\begin{array}{cc}
\boldsymbol{\Gamma}^{-1} \boldsymbol{U}_{1}^{\mathrm{T}} \boldsymbol{E} \tilde{\boldsymbol{V}}_{1} \boldsymbol{W}^{-1} & \boldsymbol{V}_{1}^{\mathrm{T}} \boldsymbol{Z} \tilde{\boldsymbol{U}}_{2} \\
\boldsymbol{V}_{2}^{\mathrm{T}} \boldsymbol{Z} \tilde{\boldsymbol{U}}_{1} & \boldsymbol{V}_{2}^{\mathrm{T}} \boldsymbol{Z} \tilde{\boldsymbol{U}}_{2}
\end{array}\right)\left(\begin{array}{c}
\tilde{\boldsymbol{U}}_{1}^{T} \\
\tilde{\boldsymbol{U}}_{2}^{T}
\end{array}\right) \\
& =\boldsymbol{V}_{1} \boldsymbol{\Gamma}^{-1} \boldsymbol{U}_{1}^{\mathrm{T}} \boldsymbol{E} \tilde{\boldsymbol{V}}_{1} \boldsymbol{W}^{-1} \tilde{\boldsymbol{U}}_{1}^{\mathrm{T}}+\boldsymbol{V}_{2} \boldsymbol{V}_{2}^{\mathrm{T}} \boldsymbol{Z} \tilde{\boldsymbol{U}}_{1} \tilde{\boldsymbol{U}}_{1}^{\mathrm{T}}+\left(\boldsymbol{V}_{1} \boldsymbol{V}_{1}^{\mathrm{T}}+\boldsymbol{V}_{2} \boldsymbol{V}_{2}^{\mathrm{T}}\right) \boldsymbol{Z} \tilde{\boldsymbol{U}}_{2} \tilde{\boldsymbol{U}}_{2}^{\mathrm{T}} \\
& =\left(\begin{array}{ll}
\boldsymbol{V}_{1} & \boldsymbol{V}_{2}
\end{array}\right)\left(\begin{array}{cc}
\boldsymbol{\Gamma}^{-1} & 0 \\
0 & 0
\end{array}\right)\left(\begin{array}{c}
\boldsymbol{U}_{1}^{\mathrm{T}} \\
\boldsymbol{U}_{2}^{\mathrm{T}}
\end{array}\right) \boldsymbol{E}\left(\begin{array}{ll}
\tilde{\boldsymbol{V}}_{1} & \tilde{\boldsymbol{V}}_{2}
\end{array}\right)\left(\begin{array}{cc}
\boldsymbol{W}^{-1} & 0 \\
0 & 0
\end{array}\right)\left(\begin{array}{c}
\tilde{\boldsymbol{U}}_{1}^{\mathrm{T}} \\
\tilde{\boldsymbol{U}}_{2}^{\mathrm{T}}
\end{array}\right)+\boldsymbol{V}_{2} \boldsymbol{V}_{2}^{\mathrm{T}} \boldsymbol{Z} \tilde{\boldsymbol{U}}_{1} \tilde{\boldsymbol{U}}_{1}^{\mathrm{T}}+\boldsymbol{Z} \tilde{\boldsymbol{U}}_{2} \tilde{\boldsymbol{U}}_{2}^{\mathrm{T}} \\
& =\boldsymbol{A}^{+} \boldsymbol{E} \boldsymbol{B}^{+}+\left(\boldsymbol{I}-\boldsymbol{V}_{1} \boldsymbol{V}_{1}^{\mathrm{T}}\right) \boldsymbol{Z} \tilde{\boldsymbol{U}}_{1} \tilde{\boldsymbol{U}}_{1}^{\mathrm{T}}+\boldsymbol{Z} \tilde{\boldsymbol{U}}_{2} \tilde{\boldsymbol{U}}_{2}^{\mathrm{T}} \\
& =\boldsymbol{A}^{+} \boldsymbol{E} \boldsymbol{B}^{+}+\boldsymbol{Z}\left(\tilde{\boldsymbol{U}}_{1} \tilde{\boldsymbol{U}}_{1}^{\mathrm{T}}+\tilde{\boldsymbol{U}}_{2} \tilde{\boldsymbol{U}}_{2}^{\mathrm{T}}\right)-\boldsymbol{V}_{1} \boldsymbol{V}_{1}^{\mathrm{T}} \boldsymbol{Z} \tilde{\boldsymbol{U}}_{1} \tilde{\boldsymbol{U}}_{1}^{\mathrm{T}} \\
& =\boldsymbol{A}^{+} \boldsymbol{E} \boldsymbol{B}^{+}+\boldsymbol{Z}-\boldsymbol{A}^{+} \boldsymbol{A Z B B} \boldsymbol{B}^{+} \\
& =\boldsymbol{Z}+\boldsymbol{A}^{+}(\boldsymbol{E}-\boldsymbol{A Z \boldsymbol { B }}) \boldsymbol{B}^{+} .
\end{aligned}
$$


The theorem is proved.

Theorem 2.2. Suppose that the set $\Omega_{2}$ is nonempty. For a given $n \times n$ matrix $\mathbf{Z}$, we have

$$
P_{\Omega_{2}}(Z)=Z+C^{+}(\boldsymbol{F}-\boldsymbol{C Z D}) \boldsymbol{D}^{+} .
$$

Proof. The proof is similar to that of Theorem 2.1 and is omitted here.

For any $\boldsymbol{Z} \in R^{n \times n}$, it is easy to verify that $\boldsymbol{E}=\frac{\boldsymbol{Z}^{\mathrm{T}}+\boldsymbol{Z}}{2}$ is a symmetric matrix. Then the spectral decomposition of the matrix $E$ is

$$
\boldsymbol{E}=\boldsymbol{U} \Lambda \boldsymbol{U}^{\mathrm{T}}
$$

where $\boldsymbol{U} \boldsymbol{U}^{\mathrm{T}}=\boldsymbol{I}_{n}$ and $\Lambda=\operatorname{diag}\left(\lambda_{1}(\boldsymbol{E}), \lambda_{2}(\boldsymbol{E}), \cdots, \lambda_{n}(\boldsymbol{E})\right)$. Then by Theorem 2.1 of Higham [26] and Definition 1.1, we have

Theorem 2.3. For a given $n \times n$ matrix $\mathbf{Z}$, we have

$$
P_{\Omega_{3}}(Z)=U \operatorname{diag}\left(t_{1}, t_{2}, \cdots, t_{n}\right) U^{\mathrm{T}},
$$

where

$$
t_{i}= \begin{cases}\lambda_{i}(\boldsymbol{E}), & \lambda_{i}(\boldsymbol{E}) \geq 0 \\ 0, & \lambda_{i}(\boldsymbol{E})<0\end{cases}
$$

By Dykstra's algorithm 2.1 and noting that the projection $P_{\Omega_{1}}(\boldsymbol{Z}), P_{\Omega_{2}}(\boldsymbol{Z})$ and $P_{\Omega_{3}}(\boldsymbol{Z})$ in Theorems 2.1, 2.2 and 2.3, we get a new algorithm to compute the optimal approximate symmetric positive semidefinite solution $\hat{\boldsymbol{X}}$ of the matrix equations (1.3) which can be stated as follows.

Algorithm 2.2

1) Set the initial value $\boldsymbol{X}_{0}=\overline{\boldsymbol{X}}$;

2) Set $\boldsymbol{X}_{3}^{(0)}=\boldsymbol{X}_{0}, \quad \boldsymbol{I}_{i}^{(0)}=0, \quad i=1,2,3$;

3) For $k=1,2,3, \cdots$

$$
\boldsymbol{X}_{0}^{(k)}=\boldsymbol{X}_{3}^{(k-1)}
$$

For $i=1,2,3$

$$
\begin{gathered}
\boldsymbol{X}_{i}^{(k)}=P_{\Omega_{i}}\left(\boldsymbol{X}_{i-1}^{(k)}-\boldsymbol{I}_{i}^{(k-1)}\right), \\
\boldsymbol{I}_{i}^{(k)}=\boldsymbol{X}_{i}^{(k)}-\left(\boldsymbol{X}_{i-1}^{(k)}-\boldsymbol{I}_{i}^{(k-1)}\right)
\end{gathered}
$$

End

End

By Lemma 2.1 and (2.1), and noting that $\Omega_{1}, \Omega_{2}$ and $\Omega_{3}$ are closed convex sets, we get the convergence theorem for Algorithm 2.2.

Theorem 2.4. If the set $\Omega$ is nonempty, then the matrix sequences $\left\{X_{1}^{(k)}\right\}, \quad\left\{X_{2}^{(k)}\right\}$ and $\left\{X_{3}^{(k)}\right\}$ generated by Algorithm 2.2 converge to the projection $P_{\Omega_{1} \cap \Omega_{2} \cap \Omega_{3}}(\overline{\boldsymbol{X}})$, that is

$$
\boldsymbol{X}_{1}^{(k)} \rightarrow P_{\Omega_{1} \cap \Omega_{2} \cap \Omega_{3}}(\overline{\boldsymbol{X}}), \boldsymbol{X}_{2}^{(k)} \rightarrow P_{\Omega_{1} \cap \Omega_{2} \cap \Omega_{3}}(\overline{\boldsymbol{X}}), \boldsymbol{X}_{3}^{(k)} \rightarrow P_{\Omega_{1} \cap \Omega_{2} \cap \Omega_{3}}(\overline{\boldsymbol{X}}), k \rightarrow+\infty .
$$

Combining Theorem 2.4 and the equalities (2.3) and (2.2), we have

Theorem 2.5. If the set $\Omega$ is nonempty, then the matrix sequences $\left\{X_{1}^{(k)}\right\}, \quad\left\{X_{2}^{(k)}\right\}$ and $\left\{X_{3}^{(k)}\right\}$ generated by Algorithm 2.2 converge to optimal approximate symmetric positive semidefinite solution $\hat{\boldsymbol{X}}$ of the matrix equations (1.3). Moreover, if the initial matrix $\boldsymbol{X}_{0}=\overline{\boldsymbol{X}}=0$, then the matrix sequences $\left\{X_{1}^{(k)}\right\}$, $\left\{X_{2}^{(k)}\right\}$ and $\left\{X_{3}^{(k)}\right\}$ converge to the least Frobenius norm symmetric positive semidefinite solution of the matrix equations $\boldsymbol{A X B}=\boldsymbol{E}, \boldsymbol{C X D}=\boldsymbol{F}$.

\section{Numerical Experiments}

In this section, we give a numerical example to illustrate that the new algorithm is feasible and effective to 
compute the optimal approximate symmetric positive semidefinite solution of the matrix equation (1.3). All programs are written in M ATLAB 7.8. We denote

$$
\text { Error }=\|\boldsymbol{E}-\boldsymbol{A X} \boldsymbol{B}\|+\|\boldsymbol{F}-\boldsymbol{C X D}\|,
$$

and use the practical stopping criterion Error $\leq 1.0 \times 10^{-10}$.

Example 3.1. Consider the matrix equation (1.3) with

$$
\begin{aligned}
& \boldsymbol{A}=\left(\begin{array}{cccccc}
2 & -3 & -1 & 4 & 0 & 2 \\
-1 & -3 & 2 & 4 & -5 & 1 \\
0 & -2 & 3 & 5 & 0 & 4 \\
7 & -8 & -2 & 13 & 5 & 4 \\
-2 & 2 & -8 & -12 & -10 & 6
\end{array}\right), \quad \boldsymbol{B}=\left(\begin{array}{ccccccc}
3 & 0 & -4 & 5 & -1 & 2 & -2 \\
-2 & 3 & -4 & 2 & 4 & -1 & -3 \\
-5 & 6 & -3 & 0 & -2 & 3 & -1 \\
-2 & 3 & -1 & 3 & 0 & 5 & -2 \\
19 & -15 & -3 & 12 & 4 & -6 & -4 \\
-9 & 9 & -9 & -10 & -6 & -12 & 2
\end{array}\right), \\
& \boldsymbol{E}=\left(\begin{array}{ccccccc}
16 & 24 & -96 & 48 & -4 & -36 & -40 \\
-8 & -12 & 48 & -24 & 2 & 18 & 20 \\
20 & 30 & -120 & 60 & -5 & -45 & -50 \\
76 & 114 & -456 & 228 & -19 & -171 & -190 \\
-96 & -144 & 576 & -288 & 24 & 216 & 240
\end{array}\right), \boldsymbol{C}=\left(\begin{array}{cccccc}
1 & -1 & 3 & 4 & 0 & 7 \\
2 & -3 & 1 & 5 & -2 & 0 \\
5 & -4 & 1 & 3 & 0 & 1 \\
15 & -11 & 8 & 12 & 2 & 17 \\
31 & -29 & 8 & 30 & -6 & 5 \\
-6 & 4 & 10 & 10 & 0 & 26
\end{array}\right), \\
& \boldsymbol{D}=\left(\begin{array}{cccc}
-2 & 0 & -4 & 1 \\
-1 & 4 & -4 & 6 \\
-3 & 2 & -6 & 1 \\
-8 & 10 & -22 & 21 \\
4 & -2 & 6 & 5 \\
22 & -30 & 66 & -75
\end{array}\right), \boldsymbol{F}=\left(\begin{array}{cccc}
168 & -224 & 504 & -574 \\
36 & -48 & 108 & -123 \\
72 & -96 & 216 & -246 \\
516 & -688 & 1548 & -1763 \\
468 & -624 & 1404 & -1599 \\
528 & -704 & 1584 & -1808
\end{array}\right)
\end{aligned}
$$

Here we use ones $(n)$ and zeros $(n)$ to stand for $n \times n$ matrix of ones and zeros. It is easy to verify that $X=$ ones (5) is a solution of the matrix equations (1.4), that is to say, the set $\Omega$ is nonempty. Therefore we can use Algorithm 2.2 to compute the optimal symmetric positive semidefinite solution of the matrix equation (1.3).

1) Let $\boldsymbol{X}_{0}=\overline{\boldsymbol{X}}=\boldsymbol{I}_{6}$. After 41 iterations of Algorithm 2.2, we get the optimal approximate symmetric positive semidefinite solution

$$
\hat{\boldsymbol{X}} \approx \boldsymbol{X}_{41}=\left(\begin{array}{llllll}
1.0690 & 1.0000 & 0.7931 & 1.1379 & 0.9655 & 1.0345 \\
1.0000 & 1.0000 & 1.0000 & 1.0000 & 1.0000 & 1.0000 \\
0.7931 & 1.0000 & 1.6207 & 0.5862 & 1.1034 & 0.8966 \\
1.1379 & 1.0000 & 0.5862 & 1.2759 & 0.9310 & 1.0690 \\
0.9655 & 1.0000 & 1.1034 & 0.9310 & 1.0172 & 0.9828 \\
1.0345 & 1.0000 & 0.8966 & 1.0690 & 0.9828 & 1.0172
\end{array}\right),
$$

and its residual error

$$
\text { Error } \approx\left\|\boldsymbol{E}-\boldsymbol{A} \boldsymbol{X}_{41} \boldsymbol{B}\right\|+\left\|\boldsymbol{F}-\boldsymbol{C} \boldsymbol{X}_{41} \boldsymbol{D}\right\|=9.67 \times 10^{-10} .
$$

By concrete computations, we know that the distance from $\overline{\boldsymbol{X}}$ to the solution set $\Omega$ is

$$
\min _{\boldsymbol{X} \in \Omega}\|\boldsymbol{X}-\overline{\boldsymbol{X}}\|=\|\hat{\boldsymbol{X}}-\overline{\boldsymbol{X}}\| \approx\left\|\boldsymbol{X}_{41}-\overline{\boldsymbol{X}}\right\|=5.3852 .
$$


2) Let $\boldsymbol{X}_{0}=\overline{\boldsymbol{X}}=\left(\begin{array}{cccccc}1 & 2 & -3 & 4 & 0 & 1 \\ 2 & -2 & 3 & 5 & -6 & 4 \\ -3 & 3 & 2 & 6 & -1 & 0 \\ 4 & 5 & 6 & 5 & -3 & 2 \\ 0 & -6 & -1 & -3 & -1 & 4 \\ 1 & 4 & 0 & 2 & 4 & 0\end{array}\right)$. After 88 iterations of Algorithm 2.2, we get the optimal approximate symmetric positive semidefinite solution

$$
\hat{\boldsymbol{X}} \approx \boldsymbol{X}_{88}=\left(\begin{array}{llllll}
1.0321 & 1.0000 & 0.9037 & 1.0642 & 0.9839 & 1.0161 \\
1.0000 & 1.0000 & 1.0000 & 1.0000 & 1.0000 & 1.0000 \\
0.9037 & 1.0000 & 1.2889 & 0.8074 & 1.0482 & 0.9518 \\
1.0642 & 1.0000 & 0.8074 & 1.1284 & 0.9679 & 1.0321 \\
0.9839 & 1.0000 & 1.0482 & 0.9679 & 1.0080 & 0.9920 \\
1.0161 & 1.0000 & 0.9518 & 1.0321 & 0.9920 & 1.0080
\end{array}\right),
$$

and its residual error

$$
\text { Error } \approx\left\|\boldsymbol{E}-\boldsymbol{A} \boldsymbol{X}_{88} \boldsymbol{B}\right\|+\left\|\boldsymbol{F}-\boldsymbol{C} \boldsymbol{X}_{88} \boldsymbol{D}\right\|=9.95 \times 10^{-10} .
$$

By concrete computations, we know that the distance from $\overline{\boldsymbol{X}}$ to the solution set $\Omega$ is

$$
\min _{X \in \Omega}\|\boldsymbol{X}-\overline{\boldsymbol{X}}\|=\|\hat{\boldsymbol{X}}-\overline{\boldsymbol{X}}\| \approx\left\|\boldsymbol{X}_{88}-\overline{\boldsymbol{X}}\right\|=18.7825
$$

3) Let $\boldsymbol{X}_{0}=\overline{\boldsymbol{X}}=\operatorname{zeros}(6)$. After 116 iterations of Algorithm 2.2, we get the optimal approximate solution

$$
\hat{\boldsymbol{X}} \approx \boldsymbol{X}_{6}=\left(\begin{array}{llllll}
1.0000 & 1.0000 & 1.0000 & 1.0000 & 1.0000 & 1.0000 \\
1.0000 & 1.0000 & 1.0000 & 1.0000 & 1.0000 & 1.0000 \\
1.0000 & 1.0000 & 1.0000 & 1.0000 & 1.0000 & 1.0000 \\
1.0000 & 1.0000 & 1.0000 & 1.0000 & 1.0000 & 1.0000 \\
1.0000 & 1.0000 & 1.0000 & 1.0000 & 1.0000 & 1.0000 \\
1.0000 & 1.0000 & 1.0000 & 1.0000 & 1.0000 & 1.0000
\end{array}\right) \text {, }
$$

which is also the least Frobenius norm symmetric positive semidefinite solution of the matrix equations (1.3), and its residual error

$$
\text { Error } \approx\left\|\boldsymbol{E}-\boldsymbol{A} \boldsymbol{X}_{6} \boldsymbol{B}\right\|+\left\|\boldsymbol{F}-\boldsymbol{C} \boldsymbol{X}_{6} \boldsymbol{D}\right\|=8.76 \times 10^{-10} .
$$

By concrete computations, we know that the distance from $\overline{\boldsymbol{X}}$ to the solution set $\Omega$ is

$$
\min _{X \in \Omega}\|X-\overline{\boldsymbol{X}}\|=\|\hat{\boldsymbol{X}}-\overline{\boldsymbol{X}}\| \approx\left\|\boldsymbol{X}_{116}-\overline{\boldsymbol{X}}\right\|=6.0000 .
$$

Example 4.1 shows that Algorithm 2.2 is feasible and effective to compute the optimal approximate symmetric positive semidefinite solution of the matrix equations (1.3).

\section{Conclusion}

In this paper, we state Problem I as the minimization of a convex quadratic function over the intersection of three closed convex sets in the Hilbert space $R^{n \times n}$, then we can use Dykstra's alternating projection algorithm to compute the optimal approximate symmetric positive semidefinite solution of the matrix equations (1.3). If we choose the initial matrix $\boldsymbol{X}_{0}=0$, the least Frobenius norm symmetric positive semidefinite solution of the matrix equations (1.3) can be obtained. A numerical example show that the new algorithm is feasible and effective to compute the optimal approximate symmetric positive semidefinite solution of the matrix equations (1.3). 


\section{References}

[1] Bauschke, H.H. and Borwein, J.M. (1994) Dykstra’s Alternating Projection Algorithm for Two Sets. Journal of Approximation Theory, 79, 418-443. http://dx.doi.org/10.1006/jath.1994.1136

[2] Dai, H. and Lancaster, P. (1996) Linear Matrix Equations from an Inverse Problem of Vibration Theory. Linear Algebra and Its Applications, 246, 31-47. http://dx.doi.org/10.1016/0024-3795(94)00311-4

[3] Meng, T. (2001) Experimental Design and Decision Support. In: Leondes, C., Ed., Expert System the Technology of Knowledge Management and Decision Making for 21st Century, Volume 1, Academic Press, San Diego.

[4] Navarra, A., Odell, P.L. and Young, D.M. (2001) A Representation of the General Common Solution to the Matrix Equations $\boldsymbol{A}_{1} \boldsymbol{X} \boldsymbol{B}_{1}=\boldsymbol{C}_{1}, \quad \boldsymbol{A}_{2} \boldsymbol{X} \boldsymbol{B}_{2}=\boldsymbol{C}_{2}$ with Applications. Computers \& Mathematics with Applications, 41, 929-935. http://dx.doi.org/10.1016/S0898-1221(00)00330-8

[5] Wang, Q.W. (2004) A System of Matrix Equations over Arbitrary Regular Rings with Identity. Linear Algebra and Its Applications, 384, 44-53. http://dx.doi.org/10.1016/j.laa.2003.12.039

[6] Liao, A.P., Lei, Y. and Yuan, S.F. (2006) The Matrix Nearness Problem for Symmetric Matrices Associated with the Matrix Equations $\left[\boldsymbol{A}^{\mathrm{T}} \boldsymbol{X} \boldsymbol{A}, \boldsymbol{B}^{\mathrm{T}} \boldsymbol{X B}\right]=[\boldsymbol{C}, \boldsymbol{D}]$. Linear Algebra and Its Applications, 418, 939-954.

http://dx.doi.org/10.1016/j.laa.2006.03.032

[7] Liao, A.P. and Lei, Y. (2005) Least-Squares Solution with the Minimum-Norm for the Matrix Equations $[\boldsymbol{A X B}, \boldsymbol{G X H}]=[\boldsymbol{C}, \boldsymbol{D}]$. Computers \& Mathematics with Applications, 50, 539-549.

http://dx.doi.org/10.1016/j.camwa.2005.02.011

[8] Sheng, X.P. and Chen, G.L. (2007) A Finite Iterative Method for Solving a Pair of Linear Matrix Equation $(\boldsymbol{A X B}, \boldsymbol{C X D})=(\boldsymbol{C}, \boldsymbol{D})$. Applied Mathematics and Computation, 189, 1350-1358.

http://dx.doi.org/10.1016/j.amc.2006.12.026

[9] Ding, J., Liu, Y.J. and Ding, F. (2010) Iterative Solutions to Matrix Equation of the Form $\boldsymbol{A}_{i} \boldsymbol{X} \boldsymbol{B}_{i}=\boldsymbol{f}_{i}$. Computers \& Mathematics with Applications, 59, 3500-3507. http://dx.doi.org/10.1016/j.camwa.2010.03.041

[10] Peng, Y.X., Hu, X.Y. and Zhang, L. (2006) An Iterative Method for Symmetric Solutions and Optimal Approximation Solution of the System of Matrix Equations $\boldsymbol{A}_{1} \boldsymbol{X} \boldsymbol{B}_{1}=\boldsymbol{C}_{1}, \boldsymbol{A}_{2} \boldsymbol{X} \boldsymbol{B}_{2}=\boldsymbol{C}_{2}$. Applied Mathematics and Computation, 183, 1127-1137. http://dx.doi.org/10.1016/j.amc.2006.05.124

[11] Chen, Y.B., Peng Z.Y. and Zhou, T.J. (2010) LSQR Iterative Method Common Symmetric Solutions to Matrix Equations $\boldsymbol{A X B}=\boldsymbol{E}$ and $\boldsymbol{C X D}=\boldsymbol{F}$. Applied Mathematics and Computation, 217, 230-236. http://dx.doi.org/10.1016/j.amc.2010.05.053

[12] Cai, J. and Chen, G.L. (2009) An Iteraive Algorithm for the Least Squares Bisymmetric Solutions of the Matrix Equations $\boldsymbol{A}_{1} \boldsymbol{X} \boldsymbol{B}_{1}=\boldsymbol{C}_{1}, \boldsymbol{A}_{2} \boldsymbol{X} \boldsymbol{B}_{2}=\boldsymbol{C}_{2}$. Mathematical and Computer Modelling, 50, 1237-1244. http://dx.doi.org/10.1016/j.mcm.2009.07.004

[13] Dehghan, M. and Hajarian, M. (2008) An Iteraive Algorithm for Solving a Pair of Matrix Equations $\boldsymbol{A X B}=\boldsymbol{E}, \boldsymbol{C X D}=$ F over Generalized Centro-Symmetric Matrices. Computers \& Mathematics with Applications, 56, 3246-3260. http://dx.doi.org/10.1016/j.camwa.2008.07.031

[14] Li, F.L., Hu, X.Y. and Lei, Z. (2008) The Generalized Reflexive Solution for a Class of Matrix Equations $(\boldsymbol{A X}=\boldsymbol{B}, \boldsymbol{X} \boldsymbol{C}=\boldsymbol{D})$. Acta Mathematica Scientia, 28, 185-193. http://dx.doi.org/10.1016/S0252-9602(08)60019-3

[15] Peng, Z.H., Hu, X.Y. and Zhang, L. (2006) An Efficient Algorithm for the Least-Squares Reflexive Solution of the Matrix Equation $\boldsymbol{A}_{1} \boldsymbol{X} \boldsymbol{B}_{1}=\boldsymbol{C}_{1}, \quad \boldsymbol{A}_{2} \boldsymbol{X} \boldsymbol{B}_{2}=\boldsymbol{C}_{2}$. Applied Mathematics and Computation, 181, 988-999.

http://dx.doi.org/10.1016/j.amc.2006.01.071

[16] Yuan, S.F., Wang, Q.W. and Xiong, Z.P. (2013) Linear Parameterized Inverse Eigenvalue Problem of Bisymmetric Matrices. Linear Algebra and Its Applications, 439, 1990-2007. http://dx.doi.org/10.1016/j.laa.2013.05.026

[17] Yuan, S.F. and Liao, A.P. (2014) Least Squares Hermitian Solution of the Complex Matrix Equation $\boldsymbol{A X B}+\boldsymbol{C X D}=\boldsymbol{E} \quad$ with the Least Norm. Journal of the Franklin Institute, 351, 4978-4997. http://dx.doi.org/10.1016/j.jfranklin.2014.08.003

[18] Dykstra, R.L. (1983) An Algorithm for Restricted Least-Squares Regression. Journal of the American Statistical Association, 78, 837-842. http://dx.doi.org/10.1080/01621459.1983.10477029

[19] Von Numann, J. (1950) Functional Operators Volume II. Princeton University Press, Princeton.

[20] Cheney, W. and Goldstein, A. (1959) Proximity Maps for Convex Sets. Proceedings of the American Mathematical Society, 10, 448-450. http://dx.doi.org/10.1090/S0002-9939-1959-0105008-8 
[21] Mendoza, M., Raydan, M. and Tarazaga, P. (1998) Computing the Nearest Diagonally Dominant Matrix. Numerical Linear Algebra with Applications, 5, 461-474.

http://dx.doi.org/10.1002/(SICI)1099-1506(199811/12)5:6<461::AID-NLA141>3.0.CO;2-V

[22] Deutsch, F. (2001) Best Approximation in Inner Product Spaces. Springer, New York. http://dx.doi.org/10.1007/978-1-4684-9298-9

[23] Boyle, J.P. and Dykstra, R.L. (1986) A Method for Finding Projections onto the Intersections of Convex Sets in Hilbert Spaces. Lecture Notes in Statistics, 37, 28-47.

[24] Escalante, R. and Raydan, M. (1996) Dykstra’s Algorithm for a Constrained Least-Squares Matrix Problem. Numerical Linear Algebra with Applications, 3, 459-471. http://dx.doi.org/10.1002/(SICI)1099-1506(199611/12)3:6<459::AID-NLA82>3.0.CO;2-S

[25] Morillas, P.M. (2005) Dykstra’s Algorithm with Strategies for Projecting onto Certain Polyhedral Cones. Applied Mathematics and Computation, 167, 635-649. http://dx.doi.org/10.1016/j.amc.2004.06.136

[26] Higham, N. (1988) Computing a Nearest Symmetric Positive Semidefinite Matrix. Linear Algebra and Its Applications, 103, 103-118. http://dx.doi.org/10.1016/0024-3795(88)90223-6 